\title{
Membership and Fees 1992 Admission et cotisation
}

Membership in either the Canadian Political Science Association or the Société québécoise de science politique includes a subscription to the Canadian Journal of Political Science. There are two levels of membership fees. Income level stated refers to gross annual income (before taxes). Members may subscribe to Canadian Public Policy at a special rate.

La cotisation à l'Association canadienne de science politique ou à la Société québécoise de science politique comprend un abonnement à la Revue canadienne de science politique. Il y a deux niveaux de frais de cotisation dans chaque catégorie. Le montant de la cotisation est déterminé en fonction de votre revenu annuel brut (avant impôt). En tant que membre il vous est possible de vous abonner à un prix avantageux à L'Analyse de politiques.

The choice of Associations/

Students, retired or unemployed individuals and any other person earning under $\$ 20,000$ annually/ Étudiant(e)s, personnes retraitées ou sans travail et toutes autres Tous les personnes dont le revenu annuel autres Choix d'associations ne dépasse pas $20000 \$$

All other members/ membres

\begin{tabular}{lrr}
\hline CPSA only/ACSP seulement & $\$ 35.00$ & $\$ 95.00$ \\
SQSP seulement/only* & $\$ 35.00$ & $\$ 95.00$ \\
Joint/conjoint CPSA/ACSP-SQSP* & $\$ 45.00$ & $\$ 110.00$ \\
*These categories of membership include & & \\
$\begin{array}{l}\text { a subscription to the SQSP's journal } \\
\text { Politique. / Ces catégories d'adhésion } \\
\text { comprennent un abonnement à la revue }\end{array}$ & & \\
Politique de la SQSP. & & \\
\hline
\end{tabular}

Institutional subscription $-\$ 65.00$ in Canada; $\$ 85.00$ in member countries of OECD; $\$ 65.00$ in all other countries

Abonnement institutionnel-65 $\$$ au Canada; $85 \$$ dans les pays-membres de l'OCDE; $65 \$$ dans tout autre pays

For information, please write:/

Pour des renseignements, veuillez vous adresser à:

The Canadian Journal of Political Science/

La revue canadienne de science politique

\#205-1 Stewart Street

Ottawa, Ontario K1N 6H7 